\title{
Uma Proposta de Protocolo para a Obtenção de Variáveis Visando Estudos de Modelagem Ecológica em Sistemas Pesqueiros Fluviais da Amazônia
}

Lucirene Aguiar de SOUZA ${ }^{1}$, Carlos Edwar de Carvalho FREITAS²

\section{RESUMO}

Pesquisas sobre as pescarias amazônicas apresentam problemas nas coletas de dados que impedem ou restringem as respostas resultantes da modelagem. Este trabalho tem como objetivo propor um protocolo de pesquisa, discriminando as principais variáveis necessárias à construção de modelos para analisar a pesca na região.

PALAVRAS-CHAVE: Método de modelagem, Coleta de dados, Ecossistemas.

\section{A Protocol Proposal for obtaining Variables Having in View Studies of Ecological Modeling in Fluvial Fishery Systems of Amazonia}

\section{ABSTRACT}

Research on amazonian fisheries presents problems in data collection that impedes or restricts resultant answers from modeling. This work proposes a protocol of research, that differentiates the principal variables necessary for constructing models to evaluate fishing in the region.

KEYWORDS: Modeling method, Data collection, Ecosystems.

1 Universidade Federal do Amazonas. E-mail: lucireneaguiar@yahoo.com.br

2 Universidade Federal do Amazonas 
A modelagem consiste em um conjunto de técnicas cada vez mais usadas como ferramentas destinadas a representar quantitativamente o funcionamento de um ecossistema (Jørgensen, 1999), possibilitando a construção de cenários resultantes de alterações naturais e/ou antrópicas. As tentativas de modelar sistemas pesqueiros na região amazônica defrontaram-se com vários entraves, que impediram ou restringiram a qualidade das respostas e o poder preditivo dos modelos (Souza, 2007). Este trabalho tem como objetivo propor um protocolo de pesquisa generalizado, discriminando as principais variáveis necessárias à construção de modelos dos sistemas pesqueiros da Amazônia.

Construção do Modelo Conceitual - é formado a partir do conhecimento adquirido através de observaçōes diretas sobre o sistema.

Definição das variáveis - consiste em selecionar os componentes e os processos mais relevantes para fazer parte do modelo (Gomes \& Varriale, 2001).

Montagem do modelo - é feita definindo-se, dentre as variáveis escolhidas, os fluxos energéticos, as fontes, os pontos de armazenamento, as perdas e as interações entre os componentes do modelo.

Estabelecimento das Relações Funcionais - consiste em representar as relações entre as variáveis por meio de equaçōes matemáticas, gráficas ou lógicas.

Calibração - é um processo que pode ser feito através de determinação do conjunto de parâmetros ótimos a serem utilizados, minimizando o erro do modelo em relação aos dados.
Validação do modelo - aplicar o modelo, com os parâmetros ótimos determinados na calibração, para simular um novo conjunto de dados observados, determinando o erro entre os dados simulados e os observados.

Construção de Cenários - é a utilização das informaçōes obtidas no processo de modelagem para simulação de alteraçôes no sistema.

Para exemplificar o processo de modelagem e demonstrar a importância da estimação adequada das variáveis ligadas à dinâmica populacional e ao ciclo hidrológico foi construído um modelo de um sistema pesqueiro em um ambiente lacustre (Figura 1). Nele tanto o estoque de peixes quanto o número de pessoas da população assumem valores constantes. Estes são alimentados por fluxos de natalidade e mortalidade, que por sua vez dependem do número de indivíduos de cada grupo e das suas respectivas taxas de mortalidade ou natalidade. A taxa de mortalidade humana do sistema está representada como totalmente dependente da densidade de peixes.

A modelagem de sistemas pesqueiros requer dados que possam fornecer estimativas sobre a densidade dos indivíduos pertencentes aos principais elos tróficos que influenciam o número de peixes no ambiente. A obtenção desse parâmetro é decisivo para o cálculo da probabilidade de encontro entre predadores e presas, o que segundo o modelo de LotkaVolterra, determinará a redução dos indivíduos predados, que é fator preponderante para a dinâmica da cadeia e conseqüentemente do modelo (Lotka, 1925 e Volterra, 1928). Dessa forma, são necessários medidas de abundância desses organismos, porém, estas medidas não são freqüentemente possíveis de serem efetuadas a partir das estatísticas pesqueiras

A DE MORTALIDADE HUMANA TAXA DE NATALIDADE HUMANA
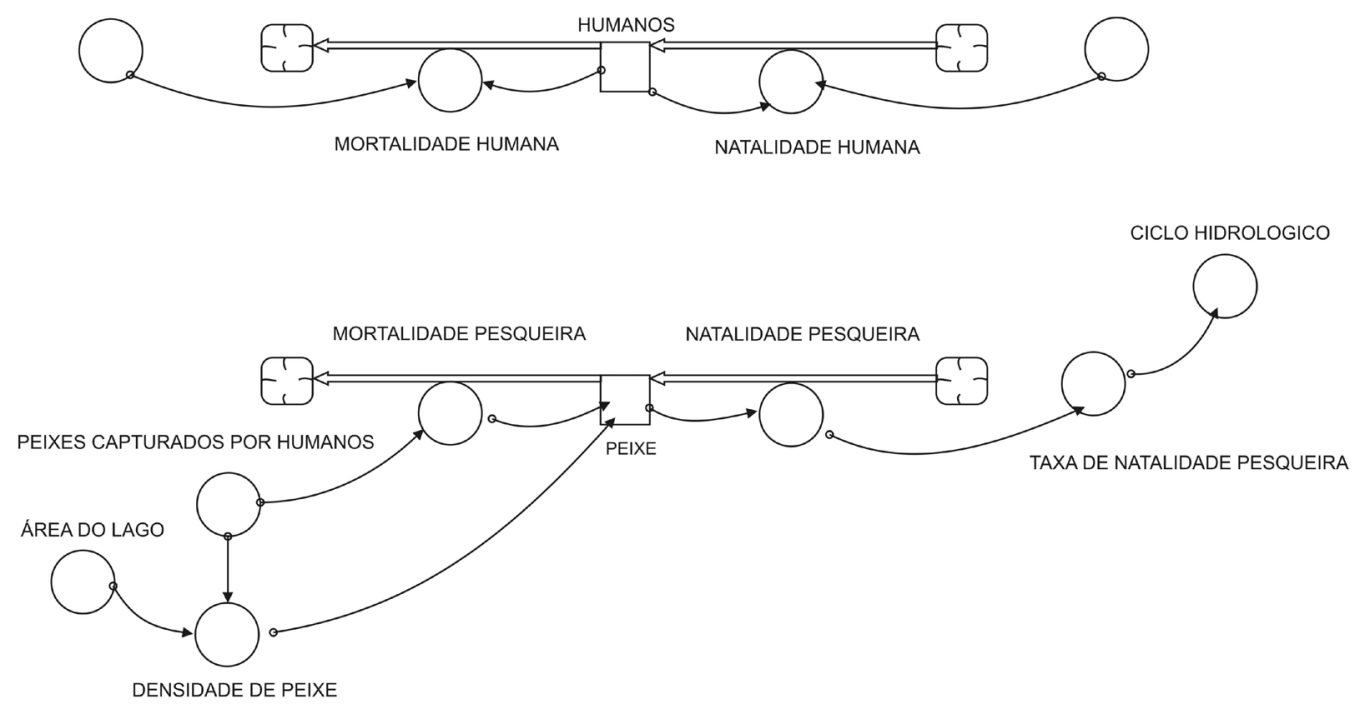

Figura 1 - Modelo de um sistema pesqueiro construído no software Stella. 
atualmente disponíveis na região. Estas são provenientes de desembarques comerciais, os quais na maioria das vezes possibilitam, quando muito, o cálculo da CPUE (Captura por Unidade de Esforço). A CPUE é um índice relativo da abundância da biomassa da população no tempo, porém ele nem sempre é um índice imparcial da abundância relativa de uma população (Seijo et al., 1997). Ele também não é muito útil quando se deseja relacionar os elos da cadeia trófica no processo de modelagem, levando em consideração a densidade de indivíduos em uma determinada área. Nesse caso a CPUA, Captura por Unidade de Área, parece ser a alternativa mais apropriada. Para obtenção desta variável é necessário o emprego de apetrechos pouco seletivos e cujo esforço nominal esteja diretamente relacionado com a área alcançada, p.ex. a rede de arrasto. Este apetrecho não é comumente usado nas pescarias desenvolvidas na Amazônia Central, dessa forma só com pescarias experimentais estes dados podem ser obtidos na região. $\mathrm{O}$ ideal seria obter os valores de abundância absoluta, que corresponde ao número de indivíduos existente por unidade de superfície ou de volume. Estes valores são de difícil determinação porque sofrem variações no tempo (flutuaçôes estacionais, anuais ou acidentais) e no espaço (de uma população para outra) (Alcântara Filho, 1988). Na maior parte dos casos nunca se consegue saber o número exato de peixes que existe numa determinada área. As razões são várias e facilmente percebidas. Para os rios da Amazônia, podemos citar que as áreas de estudo são quase sempre sistemas abertos, muito extensos e com circulação de espécies migratórias, não permitindo a realização de uma contagem efetiva. Cabe aos cientistas desenvolver métodos que contornem as dificuldades de forma que resultem em estimativas que se aproximem do número de peixes do ambiente.

É importante também coletar dados sobre abundância dos outros organismos com fortes interações com os recursos pesqueiros. Altas densidades de predadores, teoricamente, diminuem a quantidade de presas (entre elas o peixe), criando um efeito em cascata nos níveis tróficos inferiores. A quantidade mínima de presas necessária para sustentação individual de cada elo da cadeia trófica é fundamental para estimar o consumo total e, com isso, determinar o número de indivíduos necessário para manter a população em níveis sustentáveis.

Dados populacionais, como a taxa de reposição dos estoques, ainda não foram estabelecidos nem mesmo para as principais espécies comercializadas. Devido a grande taxa de mortalidade existente no primeiro período, após a eclosão dos ovos, torna-se inviável a utilização da taxa de natalidade dos peixes, sendo mais apropriada a estimação da taxa de recrutamento biológico, que refletirá com maior precisão o grau de reposição das populaçôes pesqueiras. Assim como no caso dos peixes, a taxa de reposição dos outros organismos inseridos no modelo deveria ser de alguma forma estimada.

$\mathrm{Na}$ escolha das espécies-chave, deveria se dar ênfase àquelas que são alvo da pesca comercial e que por esse motivo correm maior risco de sobre-explotação. Outro motivo relevante para eleição destas espécies é o maior interesse público, uma vez que é de interesse de todos o uso sustentável dos recursos naturais. Ainda mais privilegiadas deverão ser as espécies em regime sobrepesca, que por sua condição não podem esperar anos de coletas de dados de estatísticas pesqueiras para que se tente proceder à administração do recurso. No intuito de construir cenários que possibilitem a simulação de muitas espécies é aconselhável a coleta de dados referentes à pelo menos duas espécies de peixes, sendo que uma apresente estratégia $\mathrm{k}$ (com baixa taxa de natalidade e maturidade sexual tardia) e outra estratégia r (com alta taxa de natalidade e maturidade sexual rápida). Esse comportamento facilitaria a simulação de outras espécies com características intermediárias e, conseqüentemente, a possibilidade de avaliação destes estoques.

A inclusão do ciclo hidrológico é fundamental para construção de cenários mais realistas sobre sistemas aquáticos amazônicos, uma vez que ele é considerado a maior força controladora da biota (Junk et al., 1989). Segundo Jørgensen (1999), devemos inserir nos modelos parâmetros que possam variar de acordo com mudanças gerais no sistema. Dessa forma todos os parâmetros anteriormente citados deveriam ser medidos durante pelo menos duas fases do ciclo hidrológico, possivelmente nos valores extremos de cheia e seca.

\section{AGRADECIMENTOS}

INPA, UFAM, CNPq, PETROBRAS, FINEP e Projeto PIATAM.

\section{BIBLIOGRAFIA CITADA}

Alcântara Filho, P. 1988. Dinâmica de Populações. Parte 5. In: FAO. Manual Sobre Manejo de Reservatórios Para a Produção de Peixes. Documento de Campo 9. Documento Preparado Para o Projeto GCP/RLA/075/ITA Apoio as Atividades Regionais de Aqüicultura Para América Latina e o Caribe Programa Cooperativo Governamental. FAO - Itália.

Gomes, A. G.; Varriale, M. C., 2001. Modelagem de Ecossistemas: Uma Introdução. RS. Santa Maria. Ed da UFSM. 503pp.

Jørgensen, S.E. 1999. Recent trends in environmental and ecological modelling. An. Acad. Bras. Ci., 71: 4-11.

Junk, W. J.; Bailey, P. B.; Spark, R. E. 1989. The flood pulse concept in river floodplains systems. Can. Spec. Publ. Fish. Aquat. Sci. 106: $110-127$

Lotka A.J. 1925. Elements of Physical Biology. Williams \& Wilkens Company, Baltimore, USA. 460pp. 
Seijo, J.C.; Defeo, O.; Salas, S. 1997. Bioeconomía Pesquera: Teoría, modelación y manejo. FAO. Documento Técnico de Pesca. N. ${ }^{\circ}$ 368. Rome. FAO- Organización de las Naciones Unidas para la Agricultura y la Alimentación. 173pp.

Souza, L. A. 2007. Sustentabilidade da Pesca Através da Inclusão do Homem em Modelos Predador-Presa: Um Estudo de Caso no Lago Preto, Manacapuru, Amazonas. Tese de doutorado apresentada ao Programa de Pós-Graduação em Biologia Tropical e Recursos Naturais. INPA. 112pp.

Volterra, V. 1926. Fluctuations in the abundance of a species considered Mathematically. Nature, 118: 558-560.

Recebido em 05/06/2007

Aceito em 16/05/2008 